\title{
High-frequency absorption and gain in superlattices: Semiquasistatic approach
}

\author{
A. V. Shorokhov ${ }^{1,2}$ and K. N. Alekseev ${ }^{1}$ \\ ${ }^{1}$ Department of Physical Science, P.O. Box 3000, \\ University of Oulu FI-90014, Finland \\ ${ }^{2}$ Institute of Physics and Chemistry, \\ Mordovian State University, 430000 Saransk, Russia
}

\begin{abstract}
We consider a generation and an amplification of $\mathrm{THz}$ radiation in semiconductor superlattices under the action of microwave pump field. Electrons belonging to a single miniband of the superlattice interact quasistatically with the pump field and dynamically with a signal $\mathrm{THz}$ field. Within this semiquasistic approach we derive elegant difference formulas describing absorption (gain) of the weak $\mathrm{THz}$ signal. We present an instructive geometric interpretation of the absorption formulas which allows a search of optimum conditions for the gain employing only a simple qualitative analysis. Our theoretical findings contribute to the development of sources and detectors of $\mathrm{THz}$ radiation that are using nonlinear electric properties of semiconductor superlattices.
\end{abstract}

PACS numbers: 73.21.Cd, 07.57.Hm, 72.20.Ht

Keywords: semiconductor superlattice; Terahertz radiation; parametric generation and amplification; quasistatic interaction, quantum derivative 


\section{INTRODUCTION}

Semiconductor superlattices (SSLs) 1] have attracted growing attention in view of their unique electronic properties, which can be used for generation, amplification and detection of a high-frequency electromagnetic radiation [2, 3, 4]. A dc-biased SSL, operating in the conditions of single miniband transport regime and demonstrating the static negative differential conductance, can be potentially used as an active element of $\mathrm{THz}$ field generator [5]. However, the static negative differential conductance makes SSL unstable against a formation of high-field electric domains [6, 7]. The electric domains are believed to be destructive for the THz gain in SSLs [8]. Currently the main focus is on the possibilities to overcome this drawback within the scheme of dc-biased SSL 8, 9, 10, 11, 12]. However, schemes of THz superlattice devices with ac pump fields are also under discussion [13, 14, 15, 16]. A strong microwave field $E_{p}(t)=E_{1} \cos \left(\omega_{1} t\right)$ pumps the SSL and a desirable signal field $E_{s}(t)=E_{2} \cos \left(\omega_{2} t\right)$ has a higher frequency $\omega_{2}>\omega_{1}$. Because for typical SSLs the characteristic scattering time $\tau$ at room temperature is of the order of $100 \mathrm{fs}$, an interaction of the microwave fields with the miniband electrons is quasistatic $\left(\omega_{1} \tau \ll 1\right)$. Importantly, we have showed recently that such quasistatic pump field can completely suppress domains in SSLs [15, 16]. Two distinct possibilities exist for the signal field: It can be also quasistatic $\left(\omega_{2} \tau \ll 1\right)$ or it cannot be described within the quasistatic approach if $\omega_{2} \tau \gtrsim 1$. The later situation, i.e. $\omega_{1} \tau \ll 1$ but $\omega_{2} \tau \gtrsim 1$, can be called semiquasistatic interaction. Thus, the semiquasistatic approach is introduced to describe an amplification of $\mathrm{THz}$ field in SSL under the action of microwave field. We should note that while in the experiments [13, 14] the interaction of electrons with both pump and signal fields is still quasistatic and, moreover, it is probably domains-mediated, the ultimate goal of this activity is to reach a parametric $\mathrm{THz}$ generation without electric domains [14]. Therefore, we can say that the semiquasistatic approach describes the real experimental situations as well.

In this paper, we derive several beautiful formulas describing a high-frequency absorption (gain) of small-signal field in the presence of ac pump field, when the interaction of the

fields with the miniband electrons in the superlattice is semiquasistatic. Along with obvious potential applications in the calculations of gain in superlattice devices, our theoretical approach provides a powerful tool for finding the correspondence between quasistatic and dynamic regimes in ac-driven superlattices. 
Our main findings are following. We consider the response of electrons, belonging to a single miniband of SSL, to an action of the total electric field

$$
E(t)=E_{0}+E_{1} \cos \left(\omega_{1} t\right)+E_{2} \cos \left(\omega_{2} t\right)
$$

where $E_{0}$ is the dc bias and $E_{s}=E_{2} \cos \left(\omega_{2} t\right)$ is the weak signal (probe) field. We should note that in the real devices $E_{s}$ is a mode of the resonator tuned to a desirable $\mathrm{THz}$ frequency. Here three distinct cases should be considered: (i) $\omega_{2}$ and $\omega_{1}$ are incommensurable, (ii) $\omega_{2}=m \omega_{1} / 2$ ( $m$ is an odd number) and (iii) $\omega_{2}=m \omega_{1}$ ( $m$ is an integer). The later case of the parametric cascading is most interesting physically [16] and we mainly focus on it in this paper. We define the dimensionless absorption of the weak ac probe field in SSL as

$$
A\left(\omega_{2}\right)=2\left\langle V(t) \cos \left(\omega_{2} t\right)\right\rangle_{t}=\frac{2}{T} \int_{0}^{T} V(t) \cos \left(\omega_{2} t\right) d t
$$

where $V(t)$ is the scaled miniband electron velocity defined in the units of the maximal miniband velocity $V_{0}[1,2]$, averaging $\langle\ldots\rangle_{t}$ is performed over time and $T=2 \pi m / \omega_{2}=2 \pi / \omega_{1}$ is the common period for the probe and the pump ac fields.

Starting from the exact formal solution of the Boltzmann transport equation, we represent the absorption $A$ as the sum of three terms

$$
A=A^{h a r m}+A^{c o h}+A^{i n c o h} .
$$

Here $A^{\text {harm }}, A^{\text {coh }}$ and $A^{\text {incoh }}$ describe the absorption (gain) seeded by generation of harmonics, the parametric amplification of the probe field due to a coherent interaction of the pump and the probe fields, and the nonparametric absorption, correspondingly.

The term $A^{\text {harm }}$ is just the expression for $m$ th in-phase harmonic of the time-dependent current through SSL

$$
A^{\text {harm }}=2\left\langle I\left(U_{d c}+U_{a c} \cos \left(\omega_{1} t\right)\right) \cos \left(m \omega_{1} t\right)\right\rangle_{t},
$$

where $U_{d c}=e L E_{0}$ is the dc voltage, $L=N d$ is the length of SSL ( $d$ is the period of SSL and $N$ is the number spatial periods), $U_{a c}=e L E_{1}$ is the amplitude of ac voltage created by the pump field across SSL, the current $I(t)$ is normalized to the maximal current in SSL, $I_{0}$, corresponding to the maximal miniband velocity $V_{0}$. The explicit expressions for the $m$ th harmonic of the current, that determine the dependence $A^{\text {harm }}\left(U_{d c}, U_{a c}\right)$, are well-known 
(see [17, 18] for the case of a small pump amplitude, $U_{a c} \ll 1$, and [19, 20] for the case of an arbitrary $\left.U_{a c}\right)$. Note that $A^{\text {harm }}$ does not depend on the probe; it gives the main contribution to the absorption of a weak probe [15, 16].

The expression for $A^{\text {harm }}$ is not specific for the semiquasistic limit because of its independence on $\omega_{2}$. However, our main finding is that the absorption components $A^{\text {coh }}$ and $A^{\text {incoh }}$ can be represented within the semiquasistatic approach using the specific terms of quantum derivatives as

$A^{c o h}=e U_{2}\left\langle\frac{I^{E T}\left(U_{d c}+U_{a c} \cos \left(\omega_{1} t\right)+N \hbar \omega_{2} / e\right)-I^{E T}\left(U_{d c}+U_{a c} \cos \left(\omega_{1} t\right)-N \hbar \omega_{2} / e\right)}{2 N \hbar \omega_{2}} \cos \left(2 m \omega_{1} t\right)\right\rangle_{t}$,

$$
A^{i n c o h}=e U_{2}\left\langle\frac{I^{E T}\left(U_{d c}+U_{a c} \cos \left(\omega_{1} t\right)+N \hbar \omega_{2} / e\right)-I^{E T}\left(U_{d c}+U_{a c} \cos \left(\omega_{1} t\right)-N \hbar \omega_{2} / e\right)}{2 N \hbar \omega_{2}}\right\rangle_{t},
$$

where $U_{2}$ is the amplitude of small-signal voltage and

$$
I^{E T}(U)=\frac{U / U_{c}}{1+\left(U / U_{c}\right)^{2}}
$$

is the Esaki-Tsu voltage-current (UI) characteristic $\left(U_{c}=\hbar N / e \tau\right.$ is the critical voltage and $I^{E T}$ is normalized to the maximal current $\left.I_{0} \equiv 2 I^{E T}\left(U=U_{c}\right) \propto V_{0}\right)$.

Importantly, following Eq. (6]) in order to find the incoherent absorption in SSL at arbitrary high frequency $\omega_{2}$ we need to know only

$$
I_{d c}\left(U_{d c}\right)=\left\langle I^{E T}\left(U_{d c}+U_{a c} \cos \left(\omega_{1} t\right)\right)\right\rangle_{t}
$$

that is, the time-averaged current induced by the quasistatic field (voltage). For a given amplitude of the ac voltage $U_{a c}$, the dc current $I_{d c}$ is a function of only dc bias $U_{d c}$. It is easy to calculate or to measure the modifications of UI characteristic caused by the action of microwave (quasistatic) field [21, 22].

On the other hand, finding of the coherent component of absorption at the high frequency, which corresponds to the $m$ th harmonic of the pump frequency $\left(\omega_{2}=m \omega_{1}\right)$, is reduced following Eq. (5) to the calculation of the 2 mth harmonic of the current within the quasistatic approach. This result is especially important, because the term $A^{\text {coh }}$ can be responsible for the parametric gain in ac-driven SSLs [16]. Thus, one needs to know

$$
V_{m}^{h a r m}\left(U_{d c}\right)=2\left\langle I^{E T}\left(U_{d c}+U_{a c} \cos \left(\omega_{1} t\right)\right) \cos \left(m \omega_{1} t\right)\right\rangle_{t}
$$


(Note that in comparison with the expression (4) we have in Eq. (9): $I(U) \rightarrow I^{E T}(U)$ ). For a given pump amplitude $U_{a c}$ the amplitudes of harmonics of the quasistatic current $V_{m}^{\text {harm }}$ are functions of only dc bias $U_{d c}$. We underline that quasistatic calculations of harmonics $V_{m}^{h a r m}$ are among the standard theoretical tools to explain experimental results on the frequency multiplications in the microwave frequency band [23, 24].

In this paper we will present simple but instructive geometric interpretations of the formulas (5) and (6) allowing to find the high-frequency absorption components directly using only the ruler and the knowledge of the quasistatic curves $V_{m}^{h a r m}\left(U_{d c}\right)$ and $I_{d c}\left(U_{d c}\right)$. These geometric interpretations provide a powerful tool in a search of the optimum conditions for the gain which employs only a simple qualitative analysis.

If the frequency of the probe $\omega_{2}$ incommensurates with the frequency of the pump $\omega_{1}$, the expression for the absorption at $\omega_{2}$ consists of only one term - the incoherent absorption $A=A^{\text {incoh }}$ with semiquasistatic limit (6). Additionally, we will also show that within the semiquasistatic approach the difference formulas similar to Eqs. (15)-(6) can be written for the absorption at half-harmonics $\omega_{2}=m \omega_{1} / 2$.

The organization of this paper is as follows. We start with the calculations of absorption for arbitrary pump and probe frequencies solving the Boltzmann transport equation in sec. II] The derivation of the absorption formulas (5) and (6) within the semiquasistatic limit is presented in sec. III In the sec. IV an additional limitation on the frequency of the probe is imposed and the quasistatic formulas for the absorption are obtained. Section $\nabla$ is devoted to the presentation of useful geometric interpretations of the semiquasstatic results and their numerical verifications. The summary of the work, as well as a brief discussion of the place of our findings among other works devoted to the use of quantum derivatives in solid-state systems, are presented in the final section. Appendixes $\mathrm{A}$ and $\mathrm{B}$ are devoted respectively to the derivation of the semiquasistatic limit for the coherent component of absorption and to the derivation of the quasistatic limit for the harmonic component of absorption.

\section{ABSORPTION IN THE GENERAL CASE}

In this section we will represent the expressions for the components of absorption that follow from the exact formal solution of the transport Boltzmann equation with a constant 
relaxation time [25, 26]. Using this exact solution, we found [27] that for general case of commensurate frequencies $\omega_{1} / \omega_{2}=n / m(n, m$ are integers and $n / m$ is an irreducible fraction) the total absorption $A\left(\omega_{2}\right)$, Eq. (2) has the form

$$
\begin{aligned}
A\left(\omega_{2}\right) & =\sum_{l_{1}, l_{2}=-\infty}^{\infty} \sum_{j=-\infty}^{\infty} J_{l_{1}}\left(\beta_{1}\right) J_{l_{2}}\left(\beta_{2}\right) J_{l_{1}-j m}\left(\beta_{1}\right)\left[J_{l_{2}+j n-1}\left(\beta_{2}\right)+J_{l_{2}+j n+1}\left(\beta_{2}\right)\right] \\
& \times \frac{\left(\Omega_{0}+l_{1} \omega_{1}+l_{2} \omega_{2}\right) \tau}{1+\left(\Omega_{0}+l_{1} \omega_{1}+l_{2} \omega_{2}\right)^{2} \tau^{2}},
\end{aligned}
$$

where $\beta_{i}=\Omega_{i} / \omega_{i}, \Omega_{i}=e d E_{i} / \hbar(i=1,2)$, and $\Omega_{0}=e d E_{0} / \hbar$ is the Bloch frequency. To consider the absorption at $m$ th harmonic of the pump frequency one should fix $n=1$; for the case of absorption at half-harmonics $n$ should be equal to 2 .

First, we consider the absorption at harmonics of the pump field. In the limit of the weak probe $\left(\beta_{2} \ll 1\right)$, we need to take only certain combinations of the Bessel functions indexes. As a result we represent the absorption as a sum of three terms [27]

$$
\begin{gathered}
A=A^{\text {harm }}+A^{\text {coh }}+A^{\text {incoh }}+O\left(\beta_{2}^{2}\right) \\
A^{\text {harm }}=\sum_{l=-\infty}^{\infty} J_{l}\left(\beta_{1}\right)\left[J_{l-m}\left(\beta_{1}\right)+J_{l+m}\left(\beta_{1}\right)\right] \frac{\left(\Omega_{0}+l \omega_{1}\right) \tau}{1+\left(\Omega_{0}+l \omega_{1}\right)^{2} \tau^{2}} \\
A^{\text {coh }}=\frac{\beta_{2}}{2} \sum_{l=-\infty}^{\infty} J_{l}\left(\beta_{1}\right)\left[J_{l-2 m}\left(\beta_{1}\right)-J_{l+2 m}\left(\beta_{1}\right)\right] \frac{\left(\Omega_{0}+l \omega_{1}\right) \tau}{1+\left(\Omega_{0}+l \omega_{1}\right)^{2} \tau^{2}}, \\
A^{\text {incoh }}=\frac{\beta_{2}}{2} \sum_{l=-\infty}^{\infty} J_{l}^{2}\left(\beta_{1}\right)\left[\frac{\left(\Omega_{0}+l \omega_{1}+\omega_{2}\right) \tau}{1+\left(\Omega_{0}+l \omega_{1}+\omega_{2}\right)^{2} \tau^{2}}-\frac{\left(\Omega_{0}+l \omega_{1}-\omega_{2}\right) \tau}{1+\left(\Omega_{0}+l \omega_{1}-\omega_{2}\right)^{2} \tau^{2}}\right] .
\end{gathered}
$$

Second, for the absorption at half-harmonics of the pump frequency we get from (10) the expression for $A\left(\omega_{2}\right)$ as the sum of two terms

$$
A_{h}=A_{h}^{c o h}+A^{i n c o h}+O\left(\beta_{2}^{2}\right)
$$

where

$$
A_{h}^{c o h}=\frac{\beta_{2}}{2} \sum_{l=-\infty}^{\infty} J_{l}\left(\beta_{1}\right)\left[J_{l-m}\left(\beta_{1}\right)-J_{l+m}\left(\beta_{1}\right)\right] \frac{\left(\Omega_{0}+l \omega_{1}\right) \tau}{1+\left(\Omega_{0}+l \omega_{1}\right)^{2} \tau^{2}}
$$

and $A^{\text {incoh }}$ is given by the formula (14).

Finally, it can be shown that if $\omega_{2} / \omega_{1}$ is some irrational number, then the absorption is completely determined by the incoherent component: $A\left(\omega_{2}\right)=A^{\text {incoh }}$.

Now we turn to the consideration of semiquasistatic limits of different absorption components. 


\section{DERIVATION OF THE SEMIQUASISTATIC FORMULAS}

The derivation of semiquasistatic formulas is based on the use of the following integral representation of the Esaki-Tsu characteristic [1]

$$
I^{E T}(\omega)=\frac{\tau \omega}{1+\tau^{2} \omega^{2}} \equiv \frac{1}{\tau} \int_{0}^{\infty} \exp \left(-\frac{t}{\tau}\right) \sin (\omega t) d t .
$$

and the asymptotic saddle-point method [28].

We start with the consideration of semiquasistatic limit for the term $A^{\text {incoh }}$, Eq. (14). We rewrite $A^{\text {incoh }}$ in terms of $I^{E T}(17)$ as

$$
\begin{gathered}
A^{\text {incoh }}=\frac{\beta_{2}}{2} \sum_{l=-\infty}^{\infty} J_{l}^{2}\left(\beta_{1}\right)\left[I^{E T}\left(\Omega_{0}+l \omega_{1}+\omega_{2}\right)-I^{E T}\left(\Omega_{0}+l \omega_{1}-\omega_{2}\right)\right] \\
=\frac{\beta_{2}}{2 \tau} \int_{0}^{\infty} \exp \left(-\frac{t}{\tau}\right) \sum_{l=-\infty}^{\infty} J_{l}^{2}\left(\beta_{1}\right)\left\{\sin \left[\left(\Omega_{0}+l \omega_{1}+\omega_{2}\right) t\right]-\sin \left[\left(\Omega_{0}+l \omega_{1}-\omega_{2}\right) t\right]\right\} d t .
\end{gathered}
$$

Using the addition formula

$$
\sin \left[\left(\Omega_{0}+l \omega_{1}+\omega_{2}\right) t\right]=\sin \left[\left(\Omega_{0}+\omega_{2}\right) t\right] \cos \left(l \omega_{1} t\right)+\cos \left[\left(\Omega_{0}+\omega_{2}\right) t\right] \sin \left(l \omega_{1} t\right)
$$

and taking into account that the sum with $\sin \left(l \omega_{1} t\right)$ is equal to zero, we get

$$
\begin{aligned}
& A^{\text {incoh }}=\frac{\beta_{2}}{2 \tau} \int_{0}^{\infty} \exp \left(-\frac{t}{\tau}\right) \sum_{l=-\infty}^{\infty} J_{l}^{2}\left(\beta_{1}\right)\left\{\sin \left[\left(\Omega_{0}+l \omega_{1}+\omega_{2}\right) t\right]\right. \\
& \left.-\sin \left[\left(\Omega_{0}+l \omega_{1}-\omega_{2}\right) t\right]\right\} d t=\frac{\beta_{2}}{2 \tau} \int_{0}^{\infty} \exp \left(-\frac{t}{\tau}\right) J_{0}\left(2 \beta_{1} \sin \frac{\omega_{1} t}{2}\right) \\
& \times\left\{\sin \left[\left(\Omega_{0}+\omega_{2}\right) t\right]-\sin \left[\left(\Omega_{0}-\omega_{2}\right) t\right]\right\} d t
\end{aligned}
$$

In Eq. (18) we also have used the equality 29]

$$
\sum_{l=-\infty}^{\infty} J_{l}^{2}\left(\beta_{1}\right) \cos \left(l \omega_{1} t\right)=J_{0}\left(2 \beta_{1} \sin \frac{\omega_{1} t}{2}\right) .
$$

As a next step, we substitute the integral representation for $J_{0}(z)$ [29]

$$
J_{0}(z)=\frac{1}{\pi} \int_{0}^{\pi} \cos (z \cos \Theta) d \Theta
$$


in (18) and get

$$
\begin{gathered}
A^{\text {incoh }}=\frac{\beta_{2}}{2 \pi \tau} \int_{0}^{\infty} \exp \left(-\frac{t}{\tau}\right) \int_{0}^{\pi} \cos \left(2 \beta_{1} \sin \frac{\omega_{1} t}{2} \cos \Theta\right) d \Theta\left\{\sin \left[\left(\Omega_{0}+\omega_{2}\right) t\right]\right. \\
\left.-\sin \left[\left(\Omega_{0}-\omega_{2}\right) t\right]\right\} d t=\frac{\beta_{2}}{2 \pi \tau} \int_{0}^{\infty} \exp \left(-\frac{t}{\tau}\right) \int_{0}^{\pi}\left\{\sin \left[\left(\Omega_{1} \frac{\sin \left(\omega_{1} t / 2\right)}{\omega_{1} t / 2} \cos \Theta+\Omega_{0}+\omega_{2}\right) t\right]\right. \\
\left.-\sin \left[\left(\Omega_{1} \frac{\sin \left(\omega_{1} t / 2\right)}{\omega_{1} t / 2} \cos \Theta+\Omega_{0}-\omega_{2}\right) t\right]\right\} d \Theta d t \stackrel{\omega_{1} \tau \ll 1}{\longrightarrow} \\
\frac{\beta_{2}}{4 \pi \tau} \int_{0}^{\infty} \exp \left(-\frac{t}{\tau}\right) \int_{0}^{2 \pi}\left\{\sin \left[\left(\Omega_{1} \cos \Theta+\Omega_{0}+\omega_{2}\right) t\right]-\sin \left[\left(\Omega_{1} \cos \Theta+\Omega_{0}-\omega_{2}\right) t\right]\right\} d \Theta d t .
\end{gathered}
$$

In (19) we first used simple formula $2 \cos \alpha \sin \beta=\sin (\alpha+\beta)-\sin (\alpha-\beta)$ and then took into account that for $\omega_{1} \tau \ll 1$ the main contribution to the integrals with the exponential factor $\exp (-t / \tau)=\exp \left(-\omega_{1} t / \omega_{1} \tau\right)$ comes from the terms formally satisfying $\omega_{1} t \rightarrow 0$ (the saddle-point method). Finally, using Eq. (17) we make the inverse transformation to the Esaki-Tsu current and obtain

$$
A^{\text {incoh }}=\frac{\beta_{2}}{4 \pi} \int_{0}^{2 \pi}\left[I^{E T}\left(\Omega_{1} \cos \Theta+\Omega_{0}+\omega_{2}\right)-I^{E T}\left(\Omega_{1} \cos \Theta+\Omega_{0}-\omega_{2}\right)\right] d \Theta .
$$

Now it is easy to see that after making the transformation from frequency to voltage variables and using the Esaki-Tsu characteristic in the form (7), we get for $A^{\text {incoh }}$ the formula (6).

Applying the same method, one can obtain (see Appendix $\mathrm{A}$ ) in the semiquasistatic limit the following expression for the coherent component of absorption

$$
A^{c o h}=\frac{\beta_{2}}{4 \pi} \int_{0}^{2 \pi}\left[I^{E T}\left(\Omega_{1} \cos \Theta+\Omega_{0}+\omega_{2}\right)-I^{E T}\left(\Omega_{1} \cos \Theta+\Omega_{0}-\omega_{2}\right)\right] \cos (2 m \Theta) d \Theta,
$$

which is equivalent to the formula (5) .

We turn to the discussion of the semiquasistatic limit for absorption in the cases of incommensurable frequencies and half-harmonics. As we have found in the previous section, the absorption for incommensurable frequencies is just $A^{\text {incoh }}$ defined by Eq. (14). Therefore, its semiquasistatic limit is just the formula (20) .

Next, in the general case of arbitrary values of $\omega_{1} \tau$ and $\omega_{2} \tau$, the coherent component of absorption at half-harmonics $A_{h}^{\text {coh }}$ has the same form as the coherent component of absorption at harmonics (13), if one makes a formal change $2 m \rightarrow m$ (cf. Eqs. (16) and (13)). 
Therefore, the quasistatic limit of $A_{h}^{\text {coh }}$ coincides with Eq. (5) after the substitution $2 m \rightarrow m$. However, we should note that the consistent proof of this statement is not trivial because the derivations of semiquasistatic formulas for odd and for even values $m$ have different ways (in the case of half-harmonics $m$ is the odd number but in the case of harmonics $2 m$ is the even number).

\section{QUASISTATIC LIMIT}

To find the quasistatic limit of the components of absorption we have to impose the additional limitation on $\omega_{2}: \omega_{2} \ll \tau^{-1}$. For $A^{\text {harm }}$ we obtain from Eq. (12) in the quasistatic limit

$$
A^{\text {harm }}=\frac{1}{\pi} \int_{0}^{2 \pi} I^{E T}\left(\Omega_{0}+\Omega_{1} \cos \Theta\right) \cos (m \Theta) d \Theta .
$$

Of course, this expression is identical to Eq. (9). Formally, Eq. (22) follows from (44) after the substitution $I(U) \rightarrow I^{E T}(U)$, but the correct and consistent derivation of this result still requires some algebra (see Appendix (B)).

To get the quasistatic limit for $A^{\text {incoh }}$ and $A^{\text {coh }}$ we need to consider the limit $\omega_{2} \tau \ll 1$ in the Eqs. (201) and (21). In this limit the finite difference $I^{E T}\left(\Omega_{1} \cos \Theta+\Omega_{0}+\omega_{2}\right)-$ $I^{E T}\left(\Omega_{1} \cos \Theta+\Omega_{0}-\omega_{2}\right)$ goes into the derivative

$$
\frac{I^{E T}\left(\Omega_{1} \cos \Theta+\Omega_{0}+\omega_{2}\right)-I^{E T}\left(\Omega_{1} \cos \Theta+\Omega_{0}-\omega_{2}\right)}{2 \omega_{2}} \rightarrow \frac{\partial I^{E T}\left(\Omega_{1} \cos \Theta+\Omega_{0}\right)}{\partial \Omega_{0}}
$$

and hence $A^{\text {coh }}$ and $A^{\text {incoh }}$ become

$$
A^{c o h}=\frac{\Omega_{2}}{2 \pi} \int_{0}^{2 \pi} \frac{\partial I^{E T}\left(\Omega_{1} \cos \Theta+\Omega_{0}\right)}{\partial \Omega_{0}} \cos (2 m \Theta) d \Theta, \quad A^{i n c o h}=\frac{\Omega_{2}}{2 \pi} \int_{0}^{2 \pi} \frac{\partial I^{E T}\left(\Omega_{1} \cos \Theta+\Omega_{0}\right)}{\partial \Omega_{0}} d \Theta .
$$

These formulas in terms of voltage and current, Eq. (77), have the following form

$$
\begin{gathered}
A^{c o h}=U_{2} \frac{\partial}{\partial U_{d c}}\left\langle I^{E T}\left(U_{d c}+U_{a c} \cos \left(\omega_{1} t\right)\right) \cos \left(2 m \omega_{1} t\right)\right\rangle_{t} \\
A^{i n c o h}=U_{2} \frac{\partial}{\partial U_{d c}}\left\langle I^{E T}\left(U_{d c}+U_{a c} \cos \left(\omega_{1} t\right)\right)\right\rangle_{t} .
\end{gathered}
$$

Thus, in the quasistatic limit the incoherent and the coherent components of absorption at $m$ th harmonic are proportional respectively to the derivative of dc component (8) and to the derivative of $2 m$ th harmonic (9). 
On the other hand, the formulas for the absorption components can be derived completely within the quasistatic approach. We define the absorption of quasistatic field (voltage) as $A^{q s t}\left(\omega_{2}\right)=\left\langle I_{E T}(U) \cos \left(\omega_{2} t\right)\right\rangle_{t}$, where the Esaki-Tsu UI characteristic $I_{E T}(U)$ is given by Eq. (7). For a weak signal we expand the current in the Taylor series $I_{E T}(U) \approx I_{E T}\left(U_{p}\right)+$ $I_{E T}^{\prime}\left(U_{p}\right) \times U_{s}$ (here prime means the derivation with respect to $U, U_{p}=U_{d c}+U_{a c} \cos \left(\omega_{1} t\right)$, $\left.U_{s}=U_{2} \cos \left(\omega_{2} t\right)\right)$. Substituting this expansion to the definition of $A^{q s t}$ we have

$$
A^{q s t}\left(\omega_{2}\right)=2\left\langle I_{E T}\left(U_{p}\right) \cos \left(\omega_{2} t\right)\right\rangle_{t}+\left\langle I_{E T}^{\prime}\left(U_{p}\right) \cos \left(2 \omega_{2} t\right)\right\rangle_{t} U_{2}+\left\langle I_{E T}^{\prime}\left(U_{p}\right)\right\rangle_{t} U_{2}
$$

Making comparison of Eq. (25) with other formulas from this section, we see that in the limit $\omega_{2} \tau \rightarrow 0$ the semiquasistatic formulas for the components of absorption correctly reproduce the quasistatic results.

\section{GEOMETRICAL INTERPRETATION AND NUMERICAL VERIFICATION}

To discuss the geometric meaning of incoherent and coherent components of absorption it is convenient to present $A^{i n c o h}$ and $A^{c o h}$ explicitly in terms of zero and even harmonics of the quasistatic current $I^{E T}$. Combining (51) and (6) with (9) and (8) we have

$$
\begin{gathered}
A^{i n c o h}=\frac{e U_{2}}{2 \omega_{2}}\left[I_{d c}\left(U_{d c}+N \hbar \omega_{2} / e\right)-I_{d c}\left(U_{d c}-N \hbar \omega_{2} / e\right)\right] \\
A^{c o h}=\frac{1}{2} \frac{e U_{2}}{2 \omega_{2}}\left[V_{2 m}^{\text {harm }}\left(U_{d c}+N \hbar \omega_{2} / e\right)-V_{2 m}^{\text {harm }}\left(U_{d c}-N \hbar \omega_{2} / e\right)\right]
\end{gathered}
$$

Alternatively, the expressions for the absorption components in terms of frequency (20), (21) can be used.

As is evident from Fig. 1, the geometric representation of the quantum derivative (finite difference) of $I_{d c}$ is the segment with the length defined by the frequency of probe field $\omega_{2}$. The ends of the segment belong to the curve $I_{d c}\left(U_{d c}\right)$ and their locations are determined by the location of working point. Slope of the segment determines $A^{\text {incoh }}$ within the semiquasistatic approach. In the quasistatic limit, a finite difference becomes an usual derivative and therefore $A^{\text {incoh }}$ is determined just by the slope of the tangent line to $I_{d c}\left(U_{d c}\right)$ curve at the working point. In a similar manner, the coherent component of absorption $A^{\text {coh }}$ at the frequency $\omega_{2}=m \omega_{1}$ is determined by the slope of the segment with the ends belonging to 


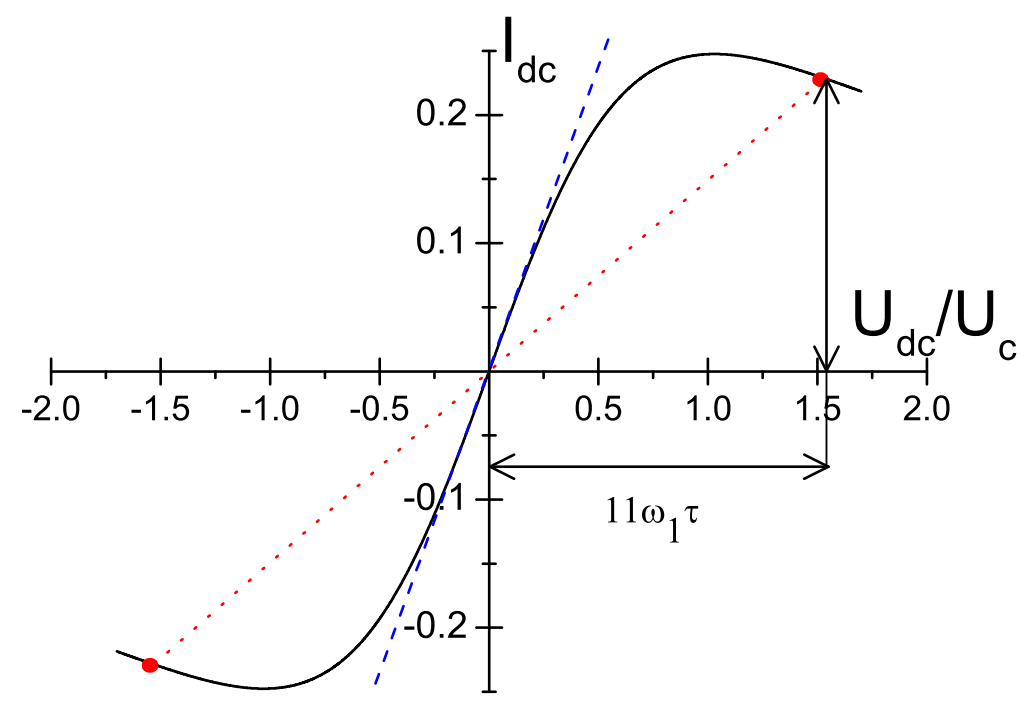

FIG. 1: [Color online] Geometrical meaning of the incoherent component of absorption within semiquasistatic and quasistatic approaches. Time-averaged current $I_{d c}$ under the action of quasistatic pump $\left(\omega_{1} \tau=0.1, U_{a c} / U_{c}=0.2\right)$ vs dc voltage $U_{d c}$. If we choose the working point at $U_{d c}=0$, then the dotted segment [red online] corresponds to the finite difference (quantum derivative) of a weak probe at $\omega_{2}=11 \omega_{1}$ and the dashed straight line [blue online] corresponds to the derivative. The slopes of these strait lines determines $A^{\text {incoh }}$ respectively in semiquasistatic and quasistatic cases.

the curve $V_{2 m}^{\text {harm }}\left(U_{d c}\right)$ (Fig. 2). Respectively, the slope of the tangent line to $V_{2 m}^{\text {harm }}\left(U_{d c}\right)$ at the working point determines $A^{\text {coh }}$ in the quasistatic limit.

Using these two geometrical pictures it is possible, in principle, to optimize a location of the working point (i.e. the value of applied dc bias) in order to obtain the maximal small-signal gain in SSL in the conditions of suppressed domains, when both $m=\omega_{2} / \omega_{1}$ and the pump amplitude $U_{a c}$ are given. However, such an analysis goes beyond the scope of the present paper. In the rest of this section we will focus on the case of dc-unbaised SSL $\left(U_{d c}=0\right)$, which corresponds to the choice of working point at the origin of coordinates in Figs. 1] and 2] Such a choice guarantees the suppression of electric domains [16]: a slope of the dependence $I_{d c}$ on $U_{d c}$ is positive practically for all $U_{a c}$ in the limit $U_{d c} \rightarrow 0$ (for examples, see Figs. 1, 4, 6).

To illustrate the usefulness of the geometric interpretation in finding of schemes allowing gain in the conditions of suppressed domains, we will consider two characteristic examples 


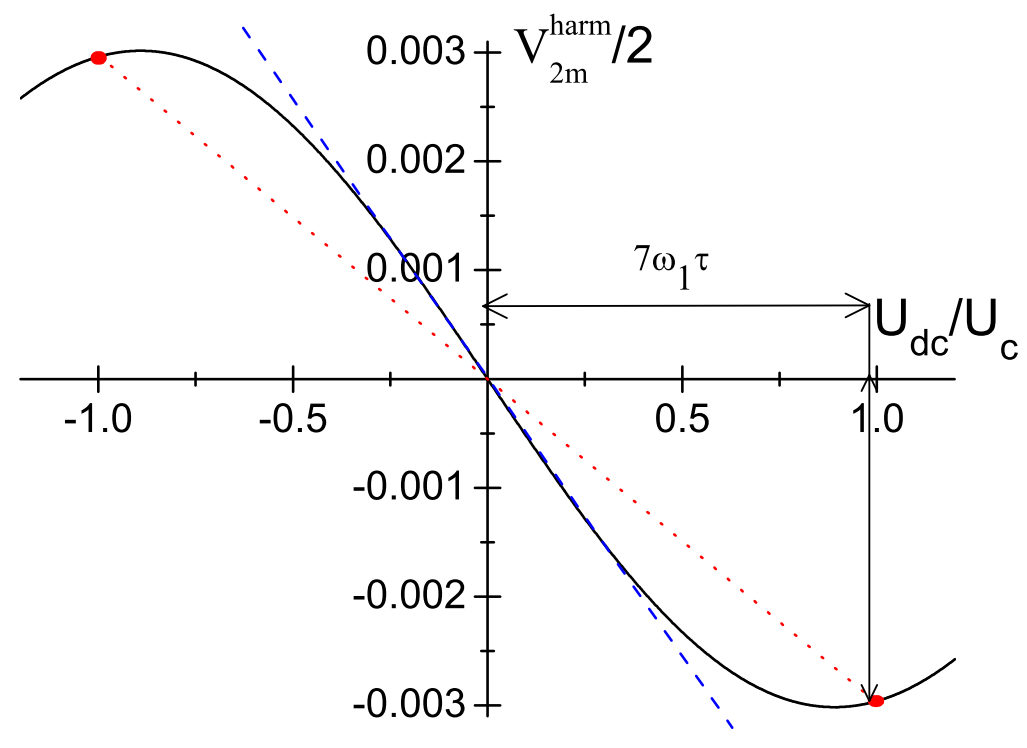

FIG. 2: [color online] Geometrical meaning of the coherent component of absorption $A^{\text {coh }}$ within semiquasistatic and quasistatic approaches. The $2 m$ th harmonic of quasistatic current $V_{2 m}^{\text {harm }}$ vs dc voltage $U_{d c}$ for $m=7$. The current is induced by the quasistatic pump $\left(\omega_{1} \tau=0.14\right)$ with the amplitude $U_{a c} / U_{c}=8$. Slope of dotted segment [red online] determines the (negative) finite difference for a weak probe with $\omega_{2}=7 \omega_{1}$. Slope of dashed straight line [blue online] determines the derivative. Working point is chosen at $U_{d c}=0$.

(earlier the solutions of these problems have been announced without proofs in [16]).

Problem I. Suppose that $\omega_{2} / \omega_{1}$ is an irrational number and therefore only the incoherent absorption $A^{\text {incoh }}$ contributes to the gain in SSL. Employing the geometrical interpretation of $A^{\text {incoh }}$, we immediately see that if the slope of the tangent line to $I_{d c}$ curve is positive, then the quantum derivative is also positive for an arbitrary $\omega_{2}$ (see, e. g., Figs. 14). In physical terms, this means that a small-signal gain in the unbiased SSL is impossible in the conditions of suppressed domains, if the frequencies of pump and probe are incommensurable.

Problem II. Consider the absorption at a probe frequency that is a half-harmonic of the pump, $\omega_{2} / \omega_{1}=m / 2$. The total absorption is the sum of the incoherent component $A^{\text {incoh }}$ and the coherent absorption at half-harmonics $A_{h}^{c o h}$ (see Eq. 16). The geometric interpretation of $A_{h}^{c o h}$ is similar to the interpretation of $A^{\text {coh }}$ : One needs only to consider $V_{m}^{\text {harm }}$ as a function of $U_{d c}$ instead of a dependence $V_{2 m}^{\text {harm }}\left(U_{d c}\right)$. Obviously, the dependence 


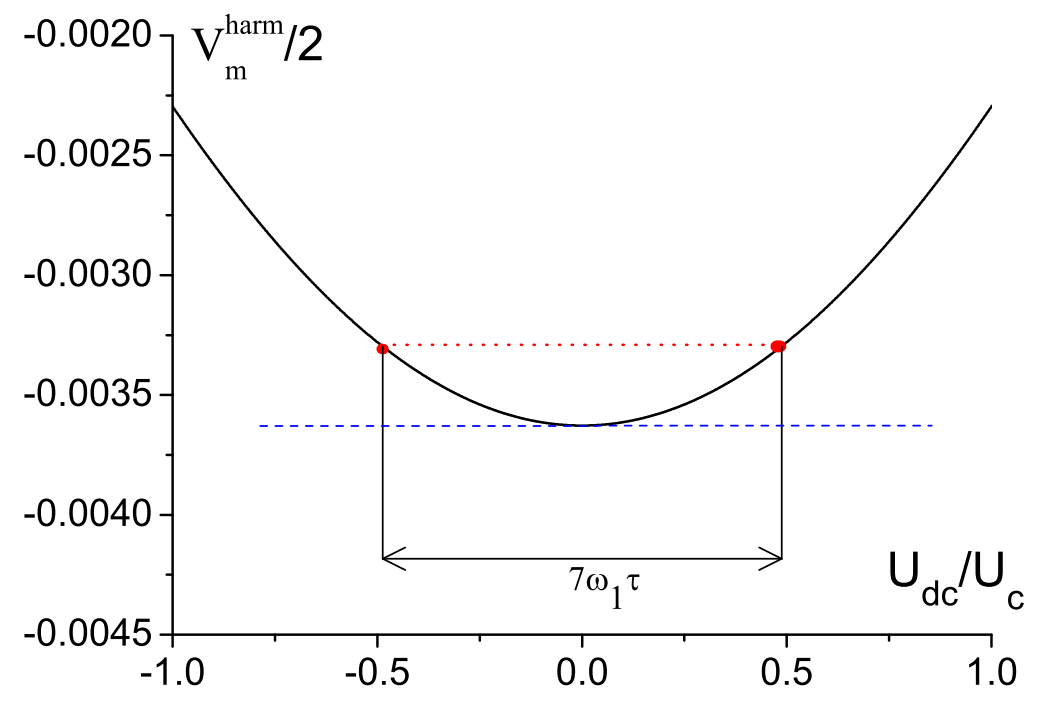

FIG. 3: [color online] Geometric interpretation for the coherent component of absorption at halfharmonics $A_{h}^{c o h}$ within semiquasistatic (dotted [red online] segment) and quasistatic (dashed [blue online] line). The curve represents $m$ th harmonic of quasistatic current $V_{m}^{\text {harm }}$ vs dc voltage $U_{d c}$ for $\omega_{2}=7 \omega_{1} / 2, \omega_{1} \tau=0.14$ and $\Omega_{1} \tau=8$. The zero slopes of the straight lines show that $A_{h}^{\text {coh }}$ calculated for $U_{d c}=0$ is zero.

of any harmonic of the quasistatic current on the applied dc bias, i. e. $V_{m}^{\text {harm }}\left(U_{d c}\right)$, is always symmetric about the vertical line $U_{d c}=0$ Therefore, the choice of the working point at $U_{d c}=0$ results in zero value of both the derivative and the quantum derivative in this point (Fig. 3). Thus, in the case of dc-unbiased SSL $A_{h}^{c o h}$ is always zero. On the other hand, as we have saw earlier, $A^{\text {incoh }}$ is always positive in the conditions of suppressed domains. Summing up we can conclude that a small-signal gain in unbiased SSL is impossible in the conditions of suppressed domains, if the frequency of probe is a half-harmonic of the pump frequency.

These two conclusive findings allow to narrow the class of possible schemes for the superlattice $\mathrm{THz}$ emitters with ac-pump, which can operate in the domainless regimes. The proper scheme should exploit a negative absorption at some particular harmonics of the probe, when negative values of harmonic $\left(A^{\text {harm }}\right)$ and coherent contributions to the net gain can overcome always positive incoherent absorption component [16]. Therefore, in our analysis we return to the case $\omega_{2} / \omega_{1}=$ integer and compare $A^{i n c o h}$ and $A^{\text {coh }}$ in conditions of 


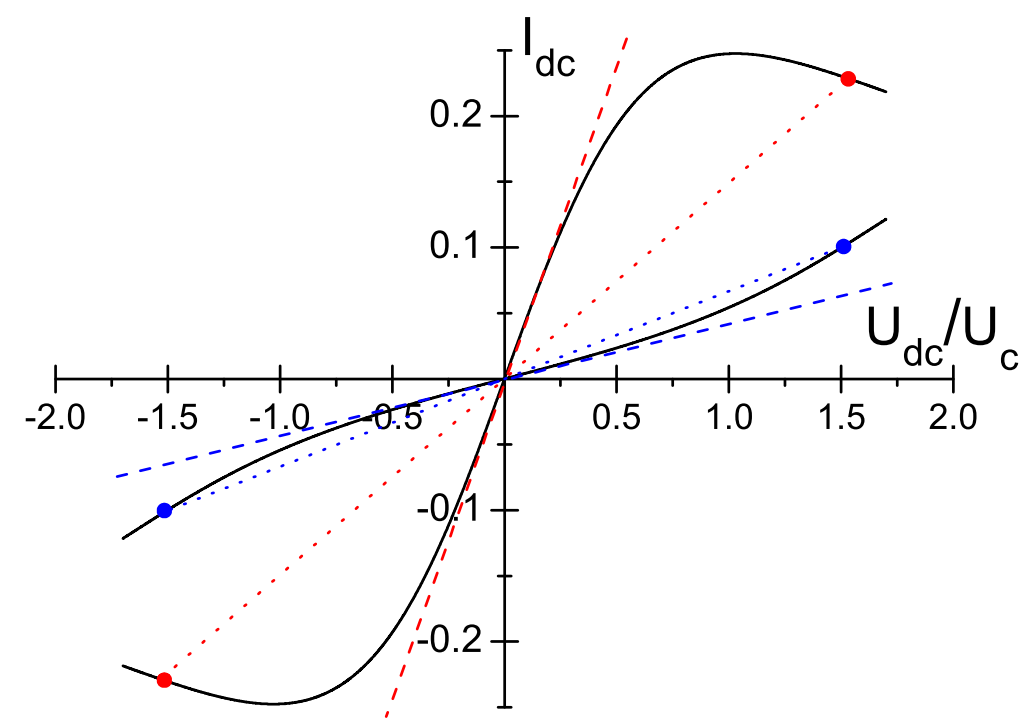

FIG. 4: [Color online] Incoherent component of absorption for different strengths of ac pump. Both difference and usual derivatives are simultaneously small and approach each other for the strong pump $\left(U_{a c} / U_{c}=2\right)$ [blue online], in contrast to the case of weak pump $\left(U_{a c} / U_{c}=0.2\right)$ [red online] where they are quite different. Here $\omega_{1} \tau=0.14$ and $m=11$.

quasistatic, semiquasistatic and dynamic approaches.

Semiquasistatic and quasistatic results have a visible difference for higher harmonics as is evident from Figs. 1] and 2 (here the quasistatic condition $\omega_{2} \tau \ll 1$ is not satisfied for the probe field). However, in the case of low-order harmonics the difference between semiquasistatic and quasistatic results is very small. This fact is illustrated in Fig. 5 for $A^{c o h}$, where the difference between the derivative (tangent line) and the quantum derivative is very small for $m=3$, but it visible increases for $m=7$.

The dependencies of $A^{\text {incoh }}$ and $A^{\text {coh }}$ on the amplitude of pump field, calculated using semiquasistatic (solid) and quasistatic (dashed) formulas for large $m$, are shown in Figs. 6 and 7. Here $A^{i n c o h}$ is always positive and its value decreases with an increase of the pump amplitude. Fig. 6] demonstrates that the quasistatic calculations significantly overestimate $A^{\text {incoh }}$ for a small pump $U_{a c} / U_{c}<1$ (see also Fig. 11). However, the agreement between semiquasistatic and quasistatic approaches in finding $A^{\text {incoh }}$ becomes good for a strong pump (Fig. 6). Such paradoxical behavior is explained by the effect of shift of the maximum of UI 


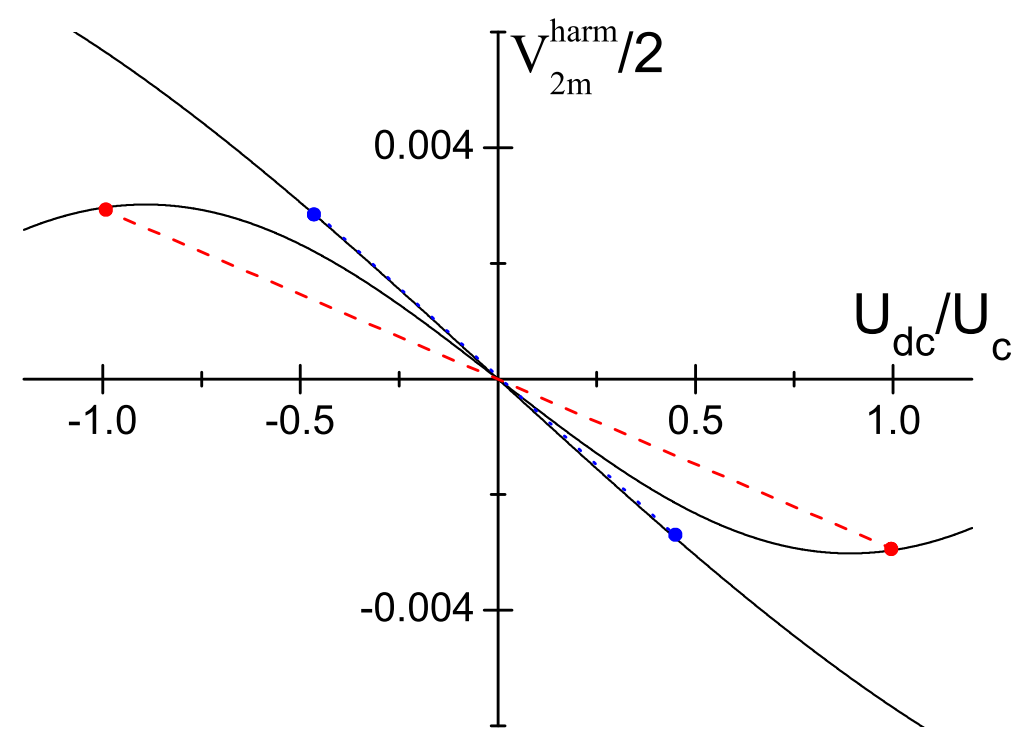

FIG. 5: [Color online] Comparison of the coherent absorptions for different values of $m=\omega_{2} / \omega_{1}$. Quantum derivative [blue online] approaches normal derivative (the tangent line, not shown) for $m=3$; they are quite different for $m=7$ [red online]. Quasistatic pump with $\omega \tau=0.14$ has the amplitude $U_{a c} / U_{c}=8$.

characteristic under the action of quasistatic ac field [30, 31] (Note: in our case the pump is still quasistatic). As we see in Fig. 4, for a large pump UI characteristic is rather closed to the linear in the interval of de voltages that is utilized for the calculation of quantum derivative, what is in a sharp contrast to the case of large ac pump where UI characteristic demonstrates a strongly nonlinear behavior in the same interval. Thus, for a strong pump the finite difference is close to the tangent line, providing a small difference between the results of semiquastatic and quasistatic approaches for $A^{i n c o h}$. As for $A^{c o h}$, the difference between semiquastatic and quasistatic calculations is quite visible for large $m$ (Fig. 7). Moreover, the quasistatic approach not only overestimate gain in this case, it can give even a wrong sign of $A^{\text {coh }}$ in some interval of ac pump amplitudes (Fig. 17).

We also calculated numerically $A^{\text {incoh }}$ and $A^{\text {incoh }}$ using formulas (13), (14) valid for arbitrary values of $\omega_{1} \tau$ and $\omega_{2} \tau$. Results of these calculations in comparison with semiquasistatic calculations are shown in Figs. 8, 9for the case of moderate pump strength and in Figs. 10, 11] for a strong ac pump. For all calculations, including those which are not shown in these 


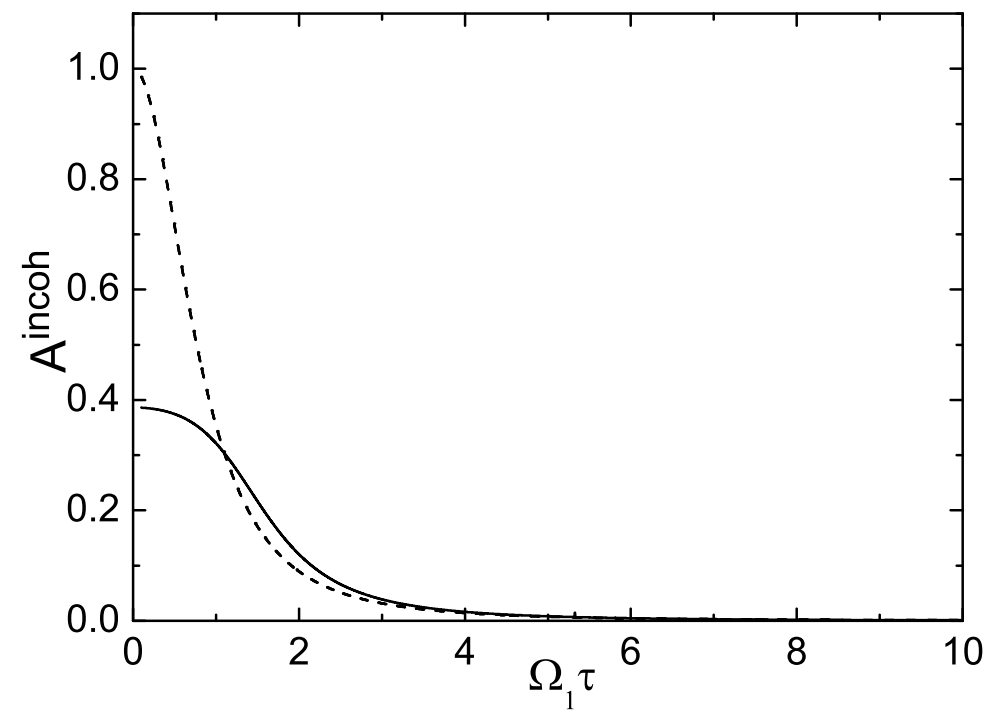

FIG. 6: Dependence of $A^{\text {incoh }}$ on the amplitude of ac pump calculated within semiquasistatic (solid) and quasistatic (dashed) approaches for $m=9$ and $\omega_{1} \tau=0.14$.

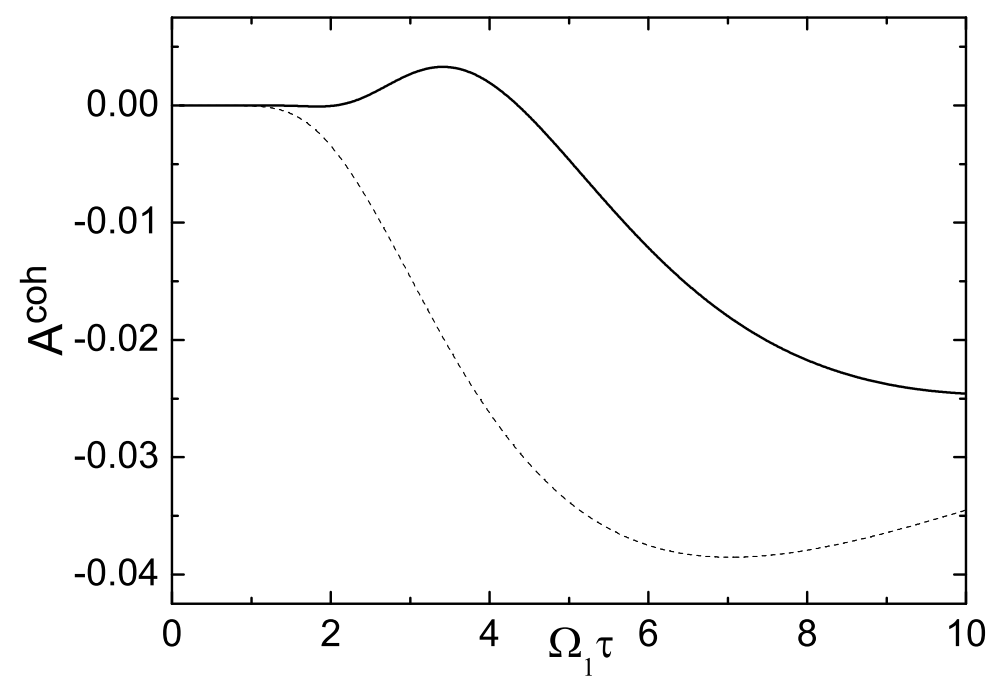

FIG. 7: Same as in Fig. 6 but for $A^{c o h}$ and $m=7$ 


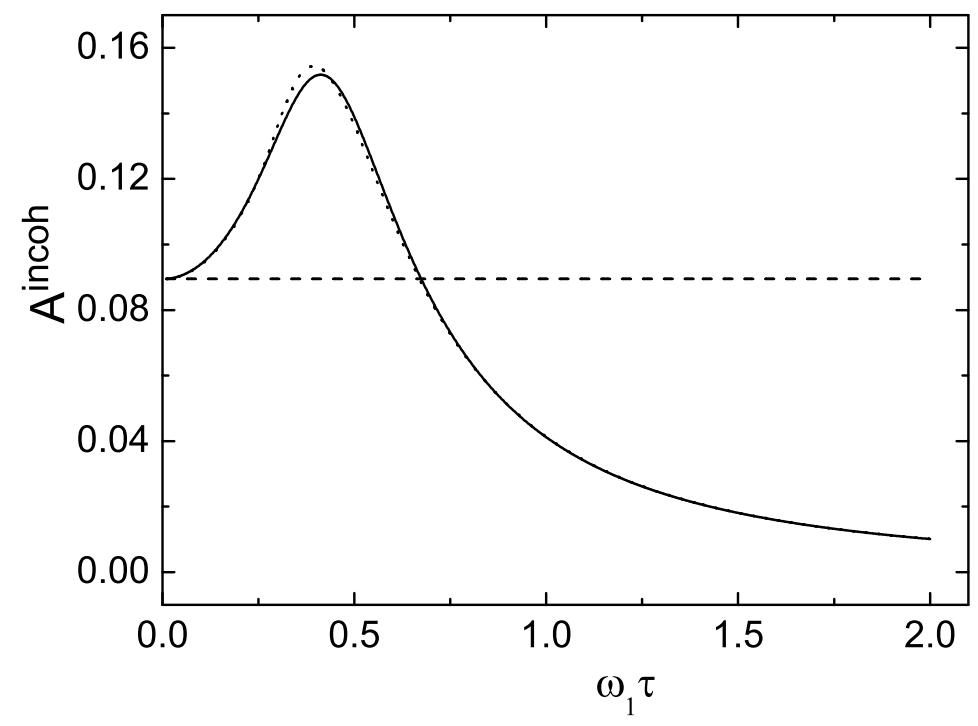

FIG. 8: Dependence of $A^{\text {incoh }}$ on $\omega_{1} \tau$ calculated within semiquasistatic (solid), dynamic (dotted) and quasistatic (dashed) approaches for $m=5$ and pump amplitude $\Omega_{1} \tau=2.0$.

figures, we always found an excellent agreement between results of dynamic (dots) and semiquasistatic (solid) approaches for small $\omega_{1} \tau$. Surprisingly, the semiquasistatic formulas work quite well even beyond their formal range of validity, i.e. not only for small values of $\omega_{1} \tau$. For the moderate pump, the semiquasistatic approach works well up to $\omega_{1} \tau \simeq 2$ (Figures 8, 91). Even for a strong enough pump, the semiquasistatic approach still gives a correct smoothed-out curve, while it does not exactly reproduce small oscillations in the dependence of absorption on the pump frequency (Figures 1011).

\section{CONCLUSION}

In summary, we calculated a small-signal absorption (gain) of a high-frequency field in semiconductor superlattice driven by a quasistatic ac field. In particular, our theoretical approach well describes practically interesting cases of generation and amplification of $\mathrm{THz}$ signal in superlattice devices pumped by a microwave field. We presented transparent geometrical interpretations for the coherent and the incoherent contributions to the net absorption at the different ratio of the pump $\left(\omega_{1}\right)$ and the signal $\left(\omega_{2}\right)$ frequencies. We 


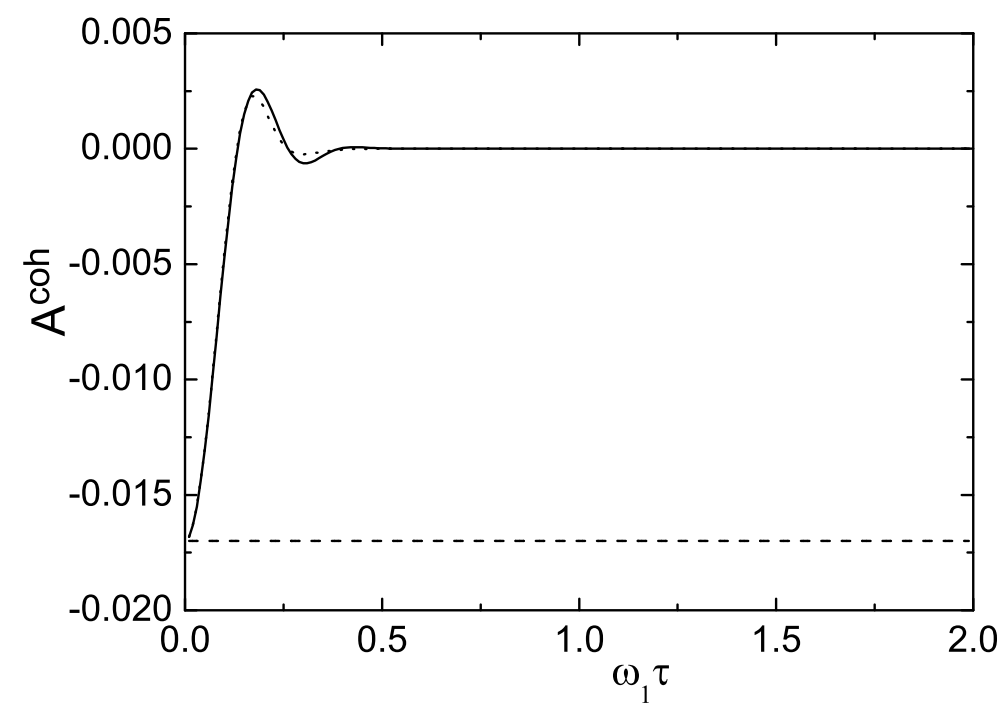

FIG. 9: Dependence of $A^{\text {coh }}$ on $\omega_{1} \tau$ calculated within semiquasistatic (solid), dynamic (dotted) and quasistatic (dashed) approaches for $m=5$ and $\Omega_{1} \tau=2.0$.

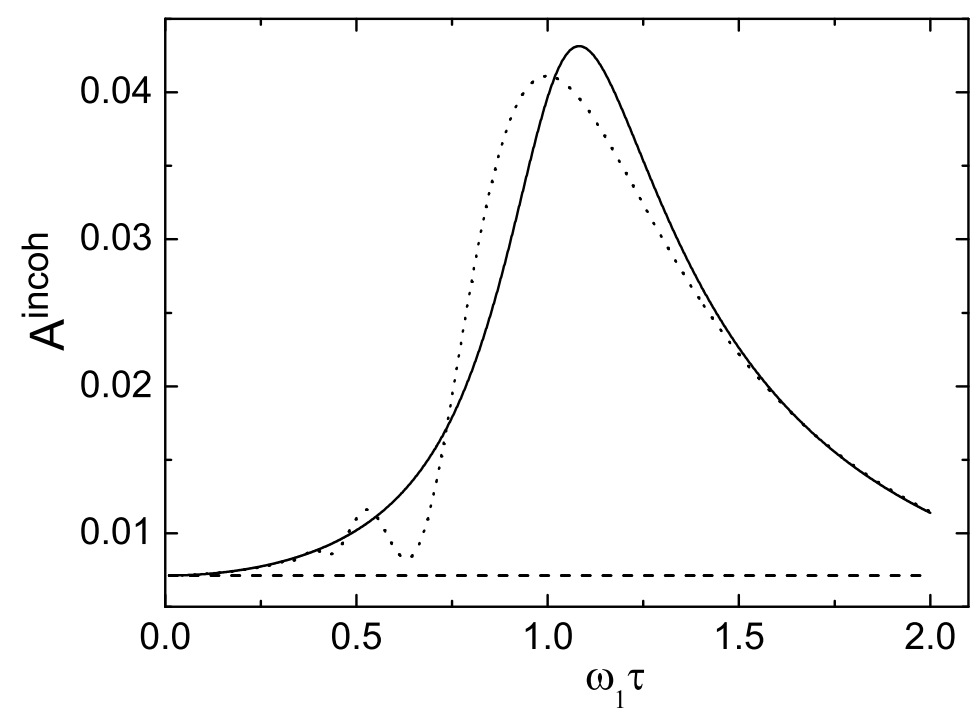

FIG. 10: Same as in Fig. 8 but for $\Omega_{1} \tau=5.1$. 


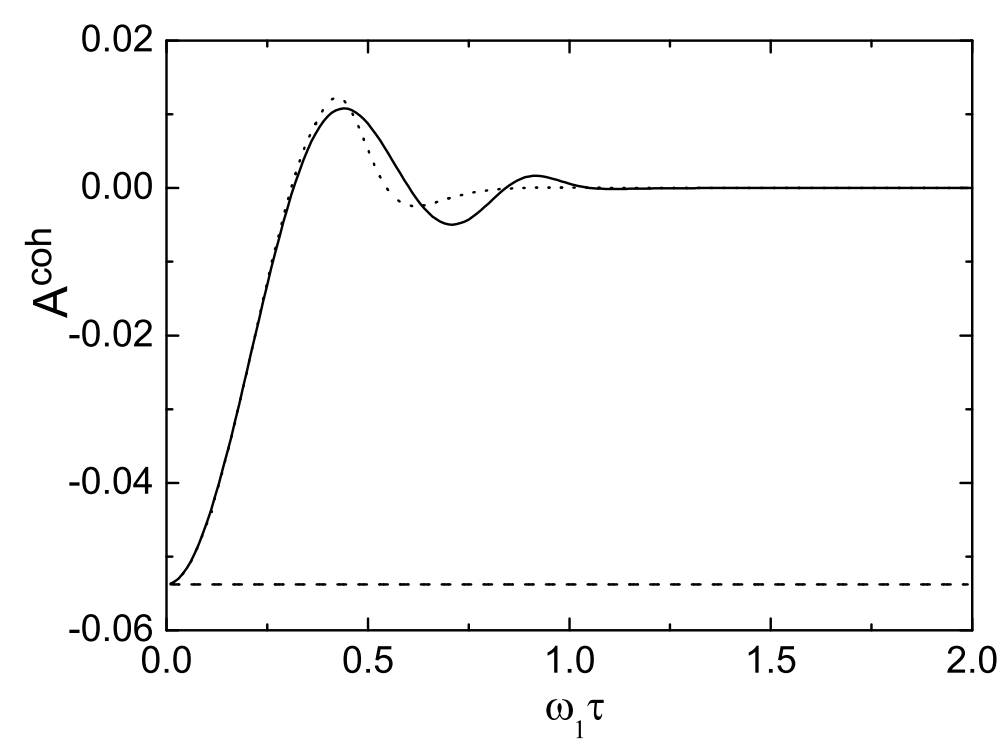

FIG. 11: Same as in Fig. 9] but for $\Omega_{1} \tau=5.1$.

demonstrated that the use of these geometric pictures sufficiently simplifies analysis: It is easy to find gain for a signal with an arbitrary $\omega_{2}$ using the electric characteristics of superlattice formed under microwave fields. In particular, we proved that for $\omega_{2} / \omega_{1}$ being some irrational number a small-signal gain at $\omega_{2}$ is possible only in the condition of static negative differential conductance. Next, we also found that an absorption at an arbitrary $\omega_{2}$, that equals to some half-integer of the pump frequency, is always zero in an unbiased superlattice. At least but not last, we presented the universal analytic procedure for finding the behavior of different physically important variables in the quasistatic limit starting from the exact solution of the Boltzmann transport equations for superlattices. Numerical calculations confirmed our analytic findings and also demonstrated that the semiquasistatic formulas for absorption work reasonable well even beyond their formal range of validity. Our results can be generalized to other physical systems where the miniband transport regime exist, such as carbon nanotubes [32] or dissipative optical lattices [33].

Although in this work we used only a single scattering time, our results obtained within semiquasistatic approximation can be also applied to a more realistic case of two different relaxation times for electron energy and electron momentum. Really, within the approximation of two relaxation times the static voltage-current characteristic of superlattice still 
has the Esaki-Tsu form [34]. Because in the semiquasistatic approach only the (quasi)static voltage-current characteristic plays a role, we speculate that a variation of the ratio of relaxation times can lead only to a some change in absolute value of the absorption but not in its dependence on the amplitude of pump field. Preliminary numerical simulations of the superlattice balance equations [19, 34] confirm these speculations [35]. The detailed consideration of $\mathrm{THz}$ absorption with the use of two different relaxation times will be published elsewhere.

It is instructive to consider our activity from the viewpoint of its place among other works devoted to the use of the quantum derivatives or, other words, to the use of the finite differences in expressions for currents. In the theory of semiconductor superlattices, the quantum derivative has been first introduced in [36] for calculations of the absorption of weak monochromatic ac field in dc-biased superlattice. Later the formula has been used in the calculations of the response function of $\mathrm{THz}$ superlattice detector [37] and in the finding of $\mathrm{THz}$ gain in the models of generalized Bloch oscillator with suppressed domains [2, 38, 39]. Interestingly, these formulas for superlattices appears to be same as the formulas describing the response of two-terminal structures to the action of dc and ac voltages [40]. The latter are widely used for calculations of signal mixing in the Josephson junctions operating in the qusiparticle transport regime and in other single-barrier tunnel devices [40, 41, 42, 43].

To the best of our knowledge this paper is the first work, where the quantum derivatives naturally appeared in a description of microstructure's response to a bichromatic field. It is an exciting problem to understand whether our semiquasistatic approach, developed for superlattices operating in the miniband transport regime, can be generalized to other tunnelling structures.

\section{Acknowledgments}

This research was supported by Academy of Finland (grants 109758) and AQDJJ Programme of European Science Foundation. We thank Timo Hyart for useful discussions and critical reading of the manuscript. 


\section{APPENDIX A: SEMIQUASISTATIC LIMIT FOR $A^{c o h}$}

In this Appendix we consider the semiquasistatic limit for $A^{\text {coh }}$. We rewrite $A^{\text {coh }}$ (13) in terms of $I^{E T}$ (17) as

$$
\begin{aligned}
A^{c o h} & =\frac{\beta_{2}}{2} \sum_{l=-\infty}^{\infty} J_{l}\left(\beta_{1}\right)\left[J_{l-2 m}\left(\beta_{1}\right)-J_{l+2 m}\left(\beta_{1}\right)\right] I^{E T}\left(\Omega_{0}+l \omega_{1}\right) \\
& =\frac{\beta_{2}}{2 \tau} \int_{0}^{\infty} \exp \left(-\frac{t}{\tau}\right) \sum_{l=-\infty}^{\infty} J_{l}\left(\beta_{1}\right)\left[J_{l-2 m}\left(\beta_{1}\right)-J_{l+2 m}\left(\beta_{1}\right)\right] \sin \left[\left(\Omega_{0}+l \omega_{1}\right) t\right] d t
\end{aligned}
$$

After changing indexes, $l \rightarrow-l$, in the first term of Eq. A1 we obtain

$$
A^{c o h}=\frac{\beta_{2}}{2 \tau} \int_{0}^{\infty} \exp \left(-\frac{t}{\tau}\right) \sum_{l=-\infty}^{\infty} J_{l}\left(\beta_{1}\right) J_{l+2 m}\left(\beta_{1}\right)\left\{\sin \left[\left(\Omega_{0}-l \omega_{1}\right) t\right]-\sin \left[\left(\Omega_{0}+l \omega_{1}\right) t\right]\right\} d t \text {. }
$$

Using the addition formula for sines we get

$$
A^{c o h}=-\frac{\beta_{2}}{\tau} \int_{0}^{\infty} \exp \left(-\frac{t}{\tau}\right) \sum_{l=-\infty}^{\infty} J_{l}\left(\beta_{1}\right) J_{l+2 m}\left(\beta_{1}\right) \sin \left(l \omega_{1} t\right) \cos \left(\Omega_{0} t\right) d t .
$$

To make the summation over $l$ we use the identity [29]

$$
\begin{aligned}
\sum_{l=-\infty}^{\infty} \sin (l a) J_{l}(x) J_{l+m}(y) & =\sin \left(m \arcsin \frac{x \sin a}{\sqrt{x^{2}+y^{2}-2 x y \cos a}}\right) \\
& \times J_{m}\left(\sqrt{x^{2}+y^{2}-2 x y \cos a}\right)
\end{aligned}
$$

and get

$$
A^{c o h}=-\frac{\beta_{2}}{\tau} \int_{0}^{\infty} \exp \left(-\frac{t}{\tau}\right) \sin \left(2 m \arcsin \cos \frac{\omega_{1} t}{2}\right) J_{2 m}\left(2 \beta_{1} \sin \frac{\omega_{1} t}{2}\right) \cos \left(\Omega_{0} t\right) d t .
$$

Now we can take into account that for $\omega_{1} \tau \ll 1$ the main contribution to the integrals with an exponential factor comes from the terms formally satisfying $\omega_{1} t \rightarrow 0$. In this case, we can use the estimations

$$
J_{2 m}\left(2 \beta_{1} \sin \frac{\omega_{1} t}{2}\right) \simeq J_{2 m}\left(\Omega_{1} t\right)
$$


because [44]

$$
\sin \left(2 m \arcsin \cos \frac{\omega_{1} t}{2}\right) \simeq(-1)^{m} \sin \left(m \omega_{1} t\right)=(-1)^{m} \sin \left(\omega_{2} t\right),
$$

$$
\arcsin (1-z)=\frac{\pi}{2}-\sqrt{2 z}, \quad \text { for } \quad|z| \ll 1 .
$$

Substituting these estimations to (A5) we derive

$$
\begin{aligned}
A^{c o h} & =-\frac{(-1)^{m} \beta_{2}}{\tau} \int_{0}^{\infty} \exp \left(-\frac{t}{\tau}\right) \sin \left(\omega_{2} t\right) \cos \left(\Omega_{0} t\right) J_{2 m}\left(\Omega_{1} t\right) d t \\
& =-\frac{(-1)^{m} \beta_{2}}{2 \tau} \int_{0}^{\infty} \exp \left(-\frac{t}{\tau}\right) J_{2 m}\left(\Omega_{1} t\right)\left[\sin \left(\omega_{2} t-\Omega_{0} t\right)+\sin \left(\omega_{2} t+\Omega_{0} t\right)\right] d t
\end{aligned}
$$

Using the integral representation for $J_{m}(z)$ [4]

$$
J_{m}(z)=\frac{1}{\pi} \int_{0}^{\pi} \cos (z \sin \Theta-m \Theta) d \Theta
$$

we have

$$
\begin{aligned}
A^{c o h} & =\frac{(-1)^{m}}{2 \pi \tau} \beta_{2} \int_{0}^{\pi} \int_{0}^{\infty} \exp \left(-\frac{t}{\tau}\right) \cos \left(\Omega_{1} t \sin \Theta-2 m \Theta\right) \\
& \times\left\{\sin \left[\left(\Omega_{0}-\omega_{2}\right) t\right]-\sin \left[\left(\Omega_{0}+\omega_{2}\right) t\right]\right\} d t d \Theta
\end{aligned}
$$

In this expression we use the formula for a product of sines and cosines and as a result we get the sum of eight integrals. These integrals are

$$
\int_{0}^{\pi} \cos \left[\Omega_{1} t \sin \Theta \pm\left(\Omega_{0}-\omega_{2}\right) t\right] \sin (2 m \Theta) d \Theta=\int_{0}^{\pi} \cos \left[\Omega_{1} t \sin \Theta \pm\left(\Omega_{0}+\omega_{2}\right) t\right] \sin (2 m \Theta) d \Theta=0 .
$$

and

$$
\begin{aligned}
& \int_{0}^{\pi}\left\{\sin \left[\Omega_{1} t \sin \Theta+\left(\Omega_{0} \mp \omega_{2}\right) t\right]-\sin \left[\Omega_{1} t \sin \Theta-\left(\Omega_{0} \mp \omega_{2}\right) t\right]\right\} \cos (2 m \Theta) d \Theta \\
= & 2(-1)^{m} \int_{0}^{\pi} \sin \left[\Omega_{1} t \cos \Theta+\left(\Omega_{0} \mp \omega_{2}\right) t\right] \cos (2 m \Theta) d \Theta .
\end{aligned}
$$

Now (A8) takes the form

$$
\begin{aligned}
A^{\text {coh }} & =-\frac{1}{2 \pi \tau} \beta_{2} \int_{0}^{\pi} \int_{0}^{\infty} \exp \left(-\frac{t}{\tau}\right)\left\{\sin \left[\Omega_{1} t \cos \Theta+\left(\Omega_{0}-\omega_{2}\right) t\right]-\sin \left[\Omega_{1} t \cos \Theta+\left(\Omega_{0}+\omega_{2}\right) t\right]\right\} \\
& \times \cos (2 m \Theta) d t d \Theta
\end{aligned}
$$


which finally allow us to make the inverse transformation by means of (17) to the Esaki-Tsu current as

$$
\begin{aligned}
A^{c o h} & =\frac{1}{4 \pi} \beta_{2} \int_{0}^{2 \pi}\left[I^{E T}\left(\Omega_{1} \cos \Theta+\Omega_{0}+\omega_{2}\right)-I^{E T}\left(\Omega_{1} \cos \Theta+\Omega_{0}-\omega_{2}\right)\right] \\
& \times \cos (2 m \Theta) d \Theta=\frac{\omega_{1}}{4 \pi} \beta_{2} \int_{0}^{2 \pi / \omega_{1}}\left[I^{E T}\left(\Omega_{1} \cos \left(\omega_{1} t\right)+\Omega_{0}+\omega_{2}\right)-I^{E T}\left(\Omega_{1} \cos \left(\omega_{1} t\right)+\Omega_{0}-\omega_{2}\right)\right] \\
& \times \cos \left(2 m \omega_{1} t\right) d t
\end{aligned}
$$

That is the desirable semiquasistatic representation for $A^{c o h}$.

\section{APPENDIX B: QUASISTATIC LIMIT FOR $A^{\text {harm }}$}

Here we consider the semiquasistatic limit for $A^{\text {harm }}$. It is convenient for our purposes to change indexes $l \rightarrow-l$ in the first term of (12) and rewrite $A^{\text {harm }}$ in the following form

$$
A^{\text {harm }}=\sum_{l=-\infty}^{\infty} J_{l}\left(\beta_{1}\right) J_{l+m}\left(\beta_{1}\right)\left[(-1)^{m} \frac{\left(\Omega_{0}-l \omega_{1}\right) \tau}{1+\left(\Omega_{0}-l \omega_{1}\right)^{2} \tau^{2}}+\frac{\left(\Omega_{0}+l \omega_{1}\right) \tau}{1+\left(\Omega_{0}+l \omega_{1}\right)^{2} \tau^{2}}\right]
$$

Now we need to rewrite $A^{\text {harm }}$ in terms of $I^{E T}$ (17) as

$$
\begin{aligned}
& A^{\text {harm }}=\sum_{l=-\infty}^{\infty} J_{l}\left(\beta_{1}\right) J_{l+m}\left(\beta_{1}\right)\left[(-1)^{m} I^{E T}\left(\Omega_{0}-l \omega_{1}\right)+I^{E T}\left(\Omega_{0}+l \omega_{1}\right)\right] \\
= & \frac{1}{\tau} \int_{0}^{\infty} \exp \left(-\frac{t}{\tau}\right) \sum_{l=-\infty}^{\infty} J_{l}\left(\beta_{1}\right) J_{l+m}\left(\beta_{1}\right)\left\{(-1)^{m} \sin \left[\left(\Omega_{0}-l \omega_{1}\right) t\right]+\sin \left[\left(\Omega_{0}+l \omega_{1}\right) t\right]\right\} d t \\
= & \frac{1}{\tau} \int_{0}^{\infty} \exp \left(-\frac{t}{\tau}\right) \sum_{l=-\infty}^{\infty} J_{l}\left(\beta_{1}\right) J_{l+m}\left(\beta_{1}\right)\left\{\left[(-1)^{m}+1\right] \sin \left(\Omega_{0} t\right) \cos \left(l \omega_{1} t\right)\right. \\
+ & {\left.\left[(-1)^{m}-1\right] \cos \left(\Omega_{0} t\right) \sin \left(l \omega_{1} t\right)\right\} d t }
\end{aligned}
$$

We can take the sum over $l$ in (B2) using Formula (A4) and the identity 29]

$$
\begin{aligned}
& \sum_{l=-\infty}^{\infty} \cos (l a) J_{l}(x) J_{l+m}(y)=\cos \left(m \arcsin \frac{x \sin a}{\sqrt{x^{2}+y^{2}-2 x y \cos a}}\right) \\
\times & J_{m}\left(\sqrt{x^{2}+y^{2}-2 x y \cos a}\right) .
\end{aligned}
$$


As a result we have

$$
\begin{aligned}
A^{\text {harm }} & =\frac{1}{\tau} \int_{0}^{\infty} \exp \left(-\frac{t}{\tau}\right)\left\{\left[(-1)^{m}+1\right] \sin \left(\Omega_{0} t\right) \cos \left(m \arcsin \cos \frac{\omega_{1} t}{2}\right) J_{m}\left(2 \beta_{1} \sin \frac{\omega_{1} t}{2}\right)\right. \\
& \left.+\left[(-1)^{m}-1\right] \cos \left(\Omega_{0} t\right) \sin \left(m \arcsin \cos \frac{\omega_{1} t}{2}\right) J_{m}\left(2 \beta_{1} \sin \frac{\omega_{1} t}{2}\right)\right\} d t .
\end{aligned}
$$

For $\omega_{1} \tau \ll 1$ the main contribution to the integrals with an exponential factor comes from the terms formally satisfying $\omega_{1} t \rightarrow 0$. Therefore, the following limits

$$
\begin{aligned}
\sin \left(m \arcsin \cos \frac{\omega_{1} t}{2}\right) & \simeq \sin \frac{m \pi}{2}, \\
\cos \left(m \arcsin \cos \frac{\omega_{1} t}{2}\right) & \simeq \cos \frac{m \pi}{2}, \\
J_{m}\left(2 \beta_{1} \sin \frac{\omega_{1} t}{2}\right) & \simeq J_{m}\left(\Omega_{1} t\right)
\end{aligned}
$$

are important. Substituting (B5) in (Bי), we obtain

$$
\begin{aligned}
A^{\text {harm }} & =\frac{1}{\tau} \int_{0}^{\infty} \exp \left(-\frac{t}{\tau}\right)\left\{\left[(-1)^{m}+1\right] \sin \left(\Omega_{0} t\right) \cos \frac{m \pi}{2} J_{m}\left(\Omega_{1} t\right)\right. \\
& \left.+\left[(-1)^{m}-1\right] \cos \left(\Omega_{0} t\right) \sin \frac{m \pi}{2} J_{m}\left(\Omega_{1} t\right)\right\} d t .
\end{aligned}
$$

Now odd and even values of $m$ must be considered separately.

$$
\begin{aligned}
& \text { If } m=2 k+1(k=0,1,2, \ldots) \text { is an odd number, then Eq. (BB6) becomes } \\
& \qquad \begin{aligned}
A^{\text {harm }} & =\frac{2(-1)^{k+1}}{\tau} \int_{0}^{\infty} \exp \left(-\frac{t}{\tau}\right) \cos \left(\Omega_{0} t\right) J_{m}\left(\Omega_{1} t\right) d t \\
& =\frac{2(-1)^{k+1}}{\pi \tau} \int_{0}^{\pi} \int_{0}^{\infty} \exp \left(-\frac{t}{\tau}\right) \cos \left(\Omega_{0} t\right) \cos \left(\Omega_{1} t \sin \Theta-m \Theta\right) d t d \Theta,
\end{aligned}
\end{aligned}
$$

where we also used the integral representation (A7) for $J_{m}$.

To simplify the expression (B7) we will need the following formula

$$
\int_{0}^{\pi} \cos \left(\Omega_{0} t\right) \cos \left(\Omega_{1} t \sin \Theta-m \Theta\right) d \Theta=(-1)^{k+1} \int_{0}^{\pi} \sin \left[\left(\Omega_{0}+\Omega_{1} \cos \Theta\right) t\right] \cos (m \Theta) d \Theta .
$$

To prove it we first convert the integral as

$$
\int_{0}^{\pi} \cos \left(\Omega_{1} t \sin \Theta-m \Theta\right) d \Theta=\int_{0}^{\pi} \sin \left(\Omega_{1} t \sin \Theta\right) \sin (m \Theta) d \Theta,
$$


and then use the formula for a product of sines and cosines

$$
2 \cos \left(\Omega_{0} t\right) \sin \left(\Omega_{1} t \sin \Theta\right)=\sin \left[\left(\Omega_{0}-\Omega_{1} \sin \Theta\right) t\right]+\sin \left[\left(\Omega_{0}+\Omega_{1} \cos \Theta\right) t\right] .
$$

Taking into account that

$$
\begin{aligned}
& \int_{0}^{\pi} \sin \left[\left(\Omega_{0}-\Omega_{1} \sin \Theta\right) t\right] \sin (m \Theta) d \Theta-\int_{0}^{\pi} \sin \left[\left(\Omega_{0}+\Omega_{1} \sin \Theta\right) t\right] \sin (m \Theta) d \Theta \\
= & 2(-1)^{k+1} \int_{0}^{\pi} \sin \left[\left(\Omega_{0}+\Omega_{1} \cos \Theta\right) t\right] \cos (m \Theta) d \Theta,
\end{aligned}
$$

we get (B8).

Now substituting (B8) in (B7) and making the inverse transformation to the Esaki-Tsu current, we obtain

$$
\begin{aligned}
A^{\text {harm }} & =\frac{2}{\pi \tau} \int_{0}^{\pi} \int_{0}^{\infty} \exp \left(-\frac{t}{\tau}\right) \sin \left[\left(\Omega_{0}+\Omega_{1} \cos \Theta\right) t\right] \cos (m \Theta) d t d \Theta \\
& =\frac{1}{\pi \tau} \int_{0}^{2 \pi} I^{E T}\left(\Omega_{0}+\Omega_{1} \cos \Theta\right) \cos (m \Theta) d \Theta=\frac{\omega_{1}}{\pi} \int_{0}^{2 \pi / \omega_{1}} I^{E T}\left(\Omega_{0}+\Omega_{1} \cos \left(\omega_{1} t\right)\right) \cos \left(m \omega_{1} t\right) d t .
\end{aligned}
$$

We turn now to the consideration of even harmonics. Combining (B6) for $m=2 k$ $(k=1,2, \ldots)$ and the integral representation (A7), we have

$$
\begin{aligned}
A^{\text {harm }} & =\frac{2(-1)^{k}}{\tau} \int_{0}^{\infty} \exp \left(-\frac{t}{\tau}\right) \sin \left(\Omega_{0} t\right) J_{m}\left(\Omega_{1} t\right) d t \\
& =\frac{2(-1)^{k}}{\pi \tau} \int_{0}^{\pi} \int_{0}^{\infty} \exp \left(-\frac{t}{\tau}\right) \sin \left(\Omega_{0} t\right) \cos \left(\Omega_{1} t \sin \Theta-m \Theta\right) d t d \Theta .
\end{aligned}
$$

Next stage of simplification includes several steps. First, we take into account that

$$
\int_{0}^{\pi} \cos \left(\Omega_{1} t \sin \Theta-m \Theta\right) d \Theta=\int_{0}^{\pi} \cos \left(\Omega_{1} t \sin \Theta\right) \cos (m \Theta) d \Theta .
$$

Second, we apply the formula for a product of sines and cosines. Finally we also use

$$
\begin{aligned}
& \int_{0}^{\pi}\left\{\sin \left[\left(\Omega_{0}+\Omega_{1} \sin \Theta\right) t\right]+\sin \left[\left(\Omega_{0}-\Omega_{1} \sin \Theta\right) t\right]\right\} \cos (m \Theta) d \Theta \\
= & 2(-1)^{k} \int_{0}^{\pi} \sin \left[\left(\Omega_{0}+\Omega_{1} \cos \Theta\right) t\right] \cos (m \Theta) d \Theta .
\end{aligned}
$$


As a result, we can represent $(\overline{B 13})$ in the form suitable for the inverse transformation to the Esaki-Tsu current and obtain

$$
\begin{aligned}
A^{\text {harm }} & =\frac{2}{\pi \tau} \int_{0}^{\pi} \int_{0}^{\infty} \exp \left(-\frac{t}{\tau}\right) \sin \left[\left(\Omega_{0}+\Omega_{1} \cos \Theta\right) t\right] \cos (m \Theta) d t d \Theta \\
& =\frac{1}{\pi} \int_{0}^{2 \pi} I^{E T}\left(\Omega_{0}+\Omega_{1} \cos \Theta\right) \cos (m \Theta) d \Theta \\
& =\frac{\omega_{1}}{\pi} \int_{0}^{2 \pi / \omega_{1}} I^{E T}\left(\Omega_{0}+\Omega_{1} \cos \left(\omega_{1} t\right)\right) \cos \left(m \omega_{1} t\right) d t
\end{aligned}
$$

The last equation coincides with the corresponding equation for odd harmonics. Thus, we conclude that in the quasistatic limit for both even and odd values of $m$

$$
A^{\text {harm }}=\frac{\omega_{1}}{\pi} \int_{0}^{2 \pi / \omega_{1}} I^{E T}\left(\Omega_{0}+\Omega_{1} \cos \left(\omega_{1} t\right)\right) \cos \left(m \omega_{1} t\right) d t .
$$

[1] L. Esaki and R. Tsu, IBM J. Res. Dev. 14 (1970) 61.

[2] A. Wacker, Phys. Rep. 357 (2002) 1.

[3] G. Platero, R. Aguado, Phys. Rep. 395 (2004) 1.

[4] A. Sibille, C. Minot, F. Laruelle, International Journal of Modern Physics B 14 (2000) 909.

[5] S. A. Ktitorov, G. S. Simin, V. Ya. Sindalovskii, Fiz. Tverd. Tela 13 (1971) 2230 [Engl. tranlation: Sov. Phys. Solid State 13 (1972) 1872 ].

[6] M. Büttiker, H. Tomas, Phys. Rev. Lett. 38 (1977) 78.

[7] A. A. Ignatov, V. I. Shashkin, Zh. Eksp. Teor. Fiz. 93 (1987) 935 [Engl. tranlation: Sov. Phys. JETP 66 (1987) 526].

[8] P. G. Savvidis, B. Kolasa, G. Lee, S. J. Allen, Phys. Rev. Lett. 92 (2004) 196802.

[9] H. Kroemer, cond-mat/0009311.

[10] T. Hyart, K. N. Alekseev, A. Leppänen, E. V. Thuneberg, cond-mat/0511145.

[11] A. Lisauskas et al., Appl. Phys. Lett. 86 (2005) 102103.

[12] T. Feil, H.-P. Tranitz, M. Reinwald, W. Wegscheider, Appl. Phys. Lett. 87 (2005) 212112. 
[13] F. Klappenberger et al., Appl. Phys. Lett. 84 (2004) 3924.

[14] K. F. Renk et al., Phys. Rev. Lett. 95 (2005) 126801.

[15] K. N. Alekseev, N. V. Demarina, M. V. Gorkunov, cond-mat/0503210.

[16] K. N. Alekseev et al., Europhys. Lett., 73 (2006) in press and cond-mat/0601056.

[17] L. Esaki, R. Tsu, Appl. Phys. Lett. 19 (1971) 246.

[18] Yu. A. Romanov, Optika Spektroskopiya 33 (1972) 917 [Engl. tranlation: Optics and Spectroscopy 33 (1972) 503].

[19] A. A. Ignatov, Yu. A. Romanov, Phys. Stat. Sol. B 73 (1976) 327.

[20] V. V. Pavlovich, E. M. Epshtein, Fiz. Tekh. Poluprovodn. 10 (1976) 2001 [Engl. tranlation: Sov. Phys. Semicond. 10 (1976) 1196].

[21] E. Shomburg et al., Appl. Phys. Lett. 68 (1996) 1096.

[22] S. Winnerl et al., Phys. Rev. B 56 (1997) 10303.

[23] J. Grenzer et al., Annalen der Physik (Leipzig) 4 (1995) 184.

[24] E. Shomburg et al., IEEE Journal of Selected Topics in Quantum Electronics 2 (1996) 724.

[25] R. G. Chambers, Proc. Phys. Soc. (London) A 65 (1952) 458.

[26] H. Budd, Phys. Rev. 127 (1962) 4.

[27] Detailed derivation of Eqs. (10)-(14) will be published elsewhere: A. V. Shorokhov, K. N. Alekseev (in preparation).

[28] M. V. Fedoryuk, The saddle-point method, (Nauka, Moscow, 1977).

[29] A. P. Prudnikov, Yu. A. Brychkov, O. I Marichev, Integrals and series, Vol. 1, (Gordon and Breach, New York, 1990).

[30] S. Y. Mensah, J. Phys. Cond. Matt. 4 (1992) L325.

[31] F. Klappenberger et al., Eur. Phys. J. B 39 (2004) 483.

[32] O. V. Kibis, D. G. Parfitt, M. E. Portnoi, Phys. Rev. B 71 (2005) 035411.

[33] G. Grynberg, C. Robilliard, Phys. Rep. 355 (2001) 335.

[34] A. A. Ignatov, E. P. Dodin, V. I. Shashkin, Mod. Phys. Lett. B 5 (1991) 1087.

[35] A. Leppänen, T. Hyart, K. N. Alekseev, Oulu university report, August 2005 (unpublished).

[36] A. A. Ignatov et al., Superlatt. Microstr. 22 (1997) 15.

[37] A. A. Ignatov, A.-P. Jauho, J. Appl. Phys. 85 (1999) 3643.

[38] A. Wacker et al., Phys. Stat. Sol. B 204 (1997) 95.

[39] T. Hyart, M. Sc. Thesis, University of Oulu, 2005. 
[40] J. R. Tucker, IEEE J. Quantum Electron. QE-15 (1979) 1234.

[41] M. J. Feldman, J. Appl. Phys. 53 (1982) 584.

[42] J. R. Tucker, M. J. Feldman, Rev. Mod. Phys. 57 (1985) 1055.

[43] A. N. Korotkov, D. V. Averiev, K. K. Likharev, Phys. Rev. B 49 (1994) 7548.

[44] M. Abramowitz, I. A. Stegun, Handbook of Mathematical Functions with Formulas, Graphs, and Mathematical Table, (Dover, New York, 1972). 